\title{
Mechanical properties of low density polymeric foams obtained from full-field measurements
}

\section{F. Pierron}

Laboratoire de Mécanique et Procédés de Fabrication, Arts et Metiers ParisTech, Rue St Dominique, B.P.508, 51006 Châlons en Champagne, France

\begin{abstract}
This exploratory paper presents some preliminary results on the use of fullfield deformation measurements on low density polymeric foams to identify the evolution of Poisson's ratio with compressive strain. Two types of foams were tested: a standard low density polyurethane foam and an auxetic foam manufactured from a similar precursor. 2D digital image correlation was used to measure the strain field at the specimens surfaces. Then, Poisson's ratios were identified using a dedicated inverse method called the Virtual Fields Method (VFM) and the results compared with the standard approaches. The results illustrate the advantages of the VFM compared to the standard procedure. It was also found that for the standard foam, very strong localization effects resulted in biased Poisson's ratio evaluation. It was shown that this could be corrected by taking into account these localization effects thanks to the full-field information.
\end{abstract}

\section{Introduction}

Low density polymeric foams are widely used in applications that require good low energy absorption capabilities, such as in packaging for instance. However, the determination of their mechanical properties is made difficult because of their hyperelastic behaviour and strong localization effects caused by the local collapse of cells in compression. The scientific literature still only provides few attempts at using full-field measurements on such foams. Some recent studies can be found but the measurements are often only used as qualitative observations of localization [1,2] or at best to validate finite element simulations [3]. It is also interesting to note that the progress in X-ray tomography has enabled several researchers to digitize the $3 \mathrm{D}$ structure of foam specimens. This can be used to build up a fully realistic 3D finite element model $[4,5]$ but more recently, first attempts at producing deformation maps have been released, either using 2D correlation on slices of 3D maps [6] or volume image correlation [7]. Poisson's ratio is an important property for foams, as detailed, for instance, in [8,9]. For low density polymeric foams, Poisson's ratio strongly varies with the strain level. The objective of the present paper is to provide some initial ideas and results on how full-field measurements can be coupled to an inverse identification procedure to identify Poisson's ratio of such low density polymeric foams. 2D digital image correlation will enable to measure the in-plane displacement field and the Virtual Fields Method (VFM, [10]) will be used to identify the material parameters.

\section{The Virtual Fields Method}

\subsection{Overview}

The Virtual Fields Method (VFM) is a dedicated tool to process full-field measurements in order to extract mechanical constitutive parameters of materials. The method relies on the principle of virtual 
work that can be written as:

$$
-\int_{V} \sigma: \epsilon^{\star} d V+\int_{S_{f}} \mathbf{T} \cdot \mathbf{u}^{\star} d S+\int_{V} \mathbf{f} \cdot \mathbf{u}^{\star} d V=\int_{V} \rho \gamma \cdot \mathbf{u}^{\star} d V
$$

where $\sigma$ is the actual stress tensor, $\epsilon^{\star}$ is the virtual strain tensor, $\mathbf{T}$ is the distribution of applied loading acting on $S_{f}, \mathbf{u}^{\star}$ is the virtual displacement, $\mathbf{f}$ is the distribution of volume forces acting on $V, \rho$ is the mass per unit volume, $\gamma$ the acceleration, '.' stands for the scalar product between two vectors and ':' stands for the contracted product between two second order tensors. An important feature is the fact that the above equation is verified for any kinematically admissible virtual field $\left(u^{*}, \epsilon^{*}\right)$. In the present case (static loading), the right-hand side term will be neglected (no inertial effects) and the volume forces (gravity) will be neglected too. The idea of the VFM is to substitute the stress tensor in Eq. 1 using the constitutive equation. In the case of linear isotropic elasticity, homogeneous material, and within the hypothesis of small deformations, this can be written as:

$$
-Q_{x x} \int_{V}\left[\varepsilon_{x} \varepsilon_{x}^{*}+\varepsilon_{y} \varepsilon_{y}^{*}+\frac{1}{2} \varepsilon_{s} \varepsilon_{s}^{*}\right] d V-Q_{x y} \int_{V}\left[\varepsilon_{x} \varepsilon_{y}^{*}+\varepsilon_{x} \varepsilon_{y}^{*}-\frac{1}{2} \varepsilon_{s} \varepsilon_{s}^{*}\right] d V+\int_{S_{f}} \mathbf{T} \cdot \mathbf{u}^{\star} d S=0
$$

The Virtual Fields Method has been applied to a number of situations within the last decade. A review article gives more details about the method [10].

\subsection{Application of the VFM to the determination of Poisson's ratio in large deformations}

The foams studied in this paper exhibit hyper-elastic behaviour, i.e. they can reach very large deformations and revert back to their initial shape after release of the load. The definition of Poisson's ratio for this situation requires some specific attention, particularly for such highly non-linear materials where Poisson's ratio is highly dependent on the strain level [11,9]. The problem was nicely addressed in [8] where the notion of Poisson's function is introduced as:

$$
v=-\frac{\Delta \varepsilon_{y}}{\Delta \varepsilon_{x}}
$$

in a uniaxial test in the $\mathrm{x}$ direction, where $\Delta \varepsilon_{y}$ and $\Delta \varepsilon_{x}$ are the strain increments between two load steps. It is equivalent to a tangent Poisson's ratio at a given deformation state. The same approach is employed here. Because Poisson's ratio is the ratio between $Q_{x y}$ and $Q_{x x}$, it is not necessary to measure a force to identify it. Indeed, if a virtual field is selected so that it is zero over the specimen's boundary where the loads are introduced, then the last term vanishes and Eq. 2 becomes:

$$
-Q_{x x} \int_{V}\left[\varepsilon_{x} \varepsilon_{x}^{*}+\varepsilon_{y} \varepsilon_{y}^{*}+\frac{1}{2} \varepsilon_{s} \varepsilon_{s}^{*}\right] d V-Q_{x y} \int_{V}\left[\varepsilon_{x} \varepsilon_{y}^{*}+\varepsilon_{x} \varepsilon_{y}^{*}-\frac{1}{2} \varepsilon_{s} \varepsilon_{s}^{*}\right] d V=0
$$

By selecting a virtual field that will ensure that the second term is not zero, then Poisson's ratio is easily given by:

$$
v=\frac{Q_{x y}}{Q_{x x}}=-\frac{\int_{V}\left[\varepsilon_{x} \varepsilon_{x}^{*}+\varepsilon_{y} \varepsilon_{y}^{*}+\frac{1}{2} \varepsilon_{s} \varepsilon_{s}^{*}\right] d V}{\int_{V}\left[\varepsilon_{x} \varepsilon_{y}^{*}+\varepsilon_{x} \varepsilon_{y}^{*}-\frac{1}{2} \varepsilon_{s} \varepsilon_{s}^{*}\right] d V}
$$

Since only surface measurements are available here, it is assumed that the strains are constant through the specimen thickness and the integrals can be integrated on $\mathrm{z}$ and the thickness cancels out in the fraction of Eq. 5. In practice, the integrals are approximated by discrete sums, for instance:

$$
\int_{S} \varepsilon_{x} \varepsilon_{x}^{*} d S \approx \sum_{i=1}^{p}\left(\varepsilon_{x}^{*}\right)^{i} \varepsilon_{x}^{i} S^{i}
$$


where $\mathrm{p}$ is the number of measurement points and $s^{i}$ the surface associated to the strain value at point i. Since in the present case, the grid of measurement points is regular, the discrete sum above becomes:

$$
\sum_{i=1}^{p}\left(\varepsilon_{x}^{*}\right)^{i} \varepsilon_{x}^{i} s^{i}=\frac{S}{p} \sum_{i=1}^{p}\left(\varepsilon_{x}^{*}\right)^{i} \varepsilon_{x}^{i}=S \overline{\varepsilon_{x}^{*} \varepsilon_{x}}
$$

where $\overline{\varepsilon_{x}^{*} \varepsilon_{x}}$ indicates the spatial averaging of function $\varepsilon_{x}^{*} \varepsilon_{x}$. Therefore, the integrals in Eq. 5 can be seen as weighted averages of the actual strains and Poisson's ratio can be rewritten as:

$$
v=-\frac{\overline{\varepsilon_{x} \varepsilon_{x}^{*}}+\overline{\varepsilon_{y} \varepsilon_{y}^{*}}+\frac{1}{2} \overline{\varepsilon_{s} \varepsilon_{s}^{*}}}{\overline{\varepsilon_{x} \varepsilon_{y}^{*}}+\overline{\varepsilon_{y} \varepsilon_{x}^{*}}-\frac{1}{2} \overline{\varepsilon_{s} \varepsilon_{s}^{*}}}
$$

\section{Test on the auxetic foam}

\subsection{Specimen manufacturing}

The specimen tested in this study is a square section cylinder of dimensions $20 \times 20 \times 85 \mathrm{~mm}$. The pristine material used to fabricate it was conventional white coloured open-cell PU based foam supplied by SM Upholstery Ltd, Cardiff, UK, with a density of $24 \mathrm{~kg} \cdot \mathrm{m}^{-3}$. A rectangular sample of $140 \times 30 \times 30 \mathrm{~mm}$ was obtained using a vertical metal cutting band saw. The sample was then converted into auxetic phase following a process similar to the one illustrated in $[12,13]$, however with a different mould geometry. More details can be found in [14].

\subsection{Longitudinal compression}

\subsubsection{Experimental description}

The first test that was performed consisted in a compression along the axis of the specimen. This was undertaken through the use of a standard clamp. Since only Poisson's ratio was sought here, no load cell was used. The CCD camera used here is a 12-bit 4000 by 2672 pixels camera. The specimen was loaded in 24 stages where pictures of the deformed specimen were taken. Some of these images can be seen on Fig. 1. The area of interest was selected on the undeformed image as shown on this figure. It consisted of 2855 by 914 pixels. Since the specimen width is $20 \mathrm{~mm}$, it gives a pixel size of about $22 \mu \mathrm{m}$. The image correlation was performed using the Correli software presented in [15], based on a fast Fourier transform algorithm. Because the specimen natural colour was yellowish, it did not provide enough contrast to perform good quality measurements and because of the very low stiffness of the foam, any type of paint would constrain the surface deformation. Therefore, the specimen surface was lightly brushed with a black felt pen to provide a greyish surface that proved much better for the correlation. A subset of $64 \times 64$ pixels with a shift of 8 pixels was selected here. Because of the very large deformations occurring in the foam, the correlation algorithm is programmed in an incremental way. Image $n$ is correlated with image $n-1$ and the deformations are cumulated in the undeformed configuration. Details of this procedure can be found in [15]. From the 24 displacement maps obtained from image correlation, the strains were calculated using a local smoothing/differentiation approach known as 'diffuse approximation', with a radius of 8 measurement points. The complete procedure is detailed in [16].

\subsubsection{Poisson's ratio identification}

The first approach to the identification of Poisson's ratio uses the common assumption of uniform uniaxial stress field. Therefore, Poisson's ratio, denoted in this case $v_{\text {uni }}$ is given as:

$$
v_{u n i}(x, y)=-\frac{\varepsilon_{y}(x, y)}{\varepsilon_{x}(x, y)}
$$




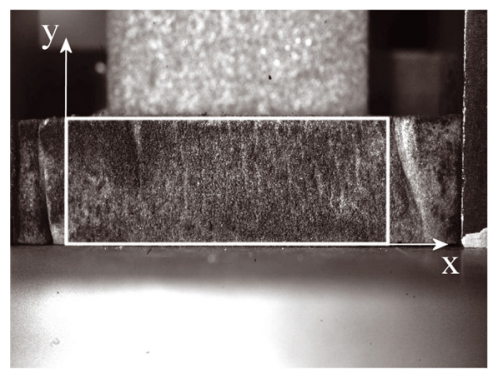

(A) Specimen at rest

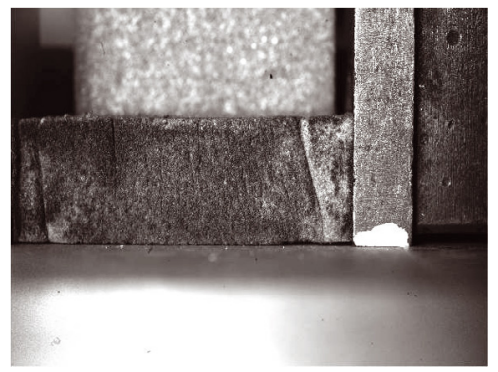

(c) Deformed specimen stage 16

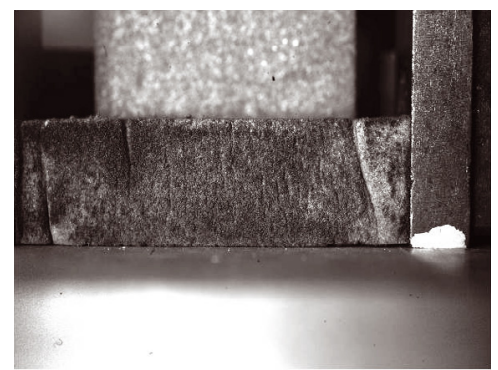

(в) Deformed specimen stage 8

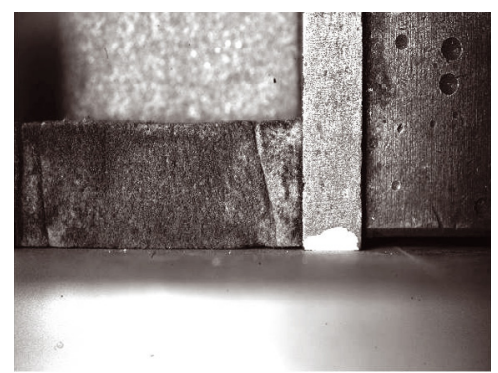

(D) Deformed specimen final stage

Fig. 1. Auxetic specimen at rest and in deformed states, longitudinal compression.

where $\varepsilon_{x}(x, y)$ is the longitudinal strain component and $\varepsilon_{y}(x, y)$ the transverse one at the point of coordinates $(x, y)$. Since strain values are available at a great number of points at the specimen surface, then an average Poisson's ratio can be defined as:

$$
v_{u n i}=-\frac{\overline{\varepsilon_{y}}}{\overline{\varepsilon_{x}}}
$$

where the upper bar indicates spatial averaging over the specimen surface. This calculation can be performed at each stage of loading. This corresponds to the values of Poisson's ratio for increasing compression levels. Instead of representing this evolution as a function of step number, it is usual to represent it as a function of the average compressive deformation, $H_{x}$ in the present case. The evolution of Poisson's ratio thus calculated is represented in Fig. 2. The evolution is very similar to that of an equivalent auxetic foam where Poisson's ratio was measured using CCD images and a contour detection algorithm [11]. The increase of Poisson's ratio towards zero describes the gradual loss of the auxetic effect by closing down of the foam cells. For the Virtual Fields Method approach, the idea is to define a virtual field that will zero the contribution of the loading forces at the ends of the specimen (see Section 2). A first simple virtual field can therefore be defined as:

$$
\left\{\begin{array}{l}
u_{x}^{*}=0 \\
u_{y}^{*}=x(x-L) y
\end{array}\right.
$$

The $x(x-L)$ term ensures that the virtual displacements are zero at both specimens edges and the $y$ term ensures a non zero transverse strain necessary for the identification since without it, only the shear strains would have been involved, which are small in this test for most load steps. The resulting equation (see Section 2) gives:

$$
v_{v f 1}=-\frac{\overline{x(x-L) \varepsilon_{y}}+\overline{\left(x-\frac{L}{2}\right) y \varepsilon_{s}}}{\overline{x(x-L) \varepsilon_{x}}-\overline{\left(x-\frac{L}{2}\right) y \varepsilon_{s}}}
$$




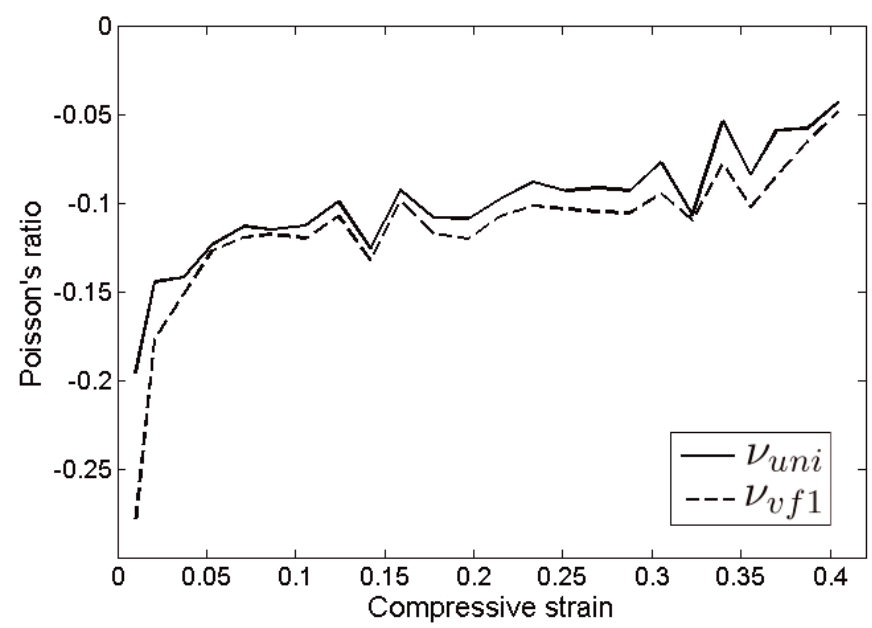

Fig. 2. Evolution of Poisson's ratio with compressive deformation, uniaxial and virtual fields approach (vf1), auxetic longitudinal compression.

The results from these calculations are plotted on Fig. 2. They are very similar to that from the uniaxial approach but significant differences are present, mainly in the early and late stages of loading. For instance, for the first load step, the uniaxial approach very significantly underestimates Poisson's ratio, with a value of -0.195 whereas the VFM approach yields a value of -0.28 . This is caused by some parasitic shear strain present in the early stages of deformation only. The Virtual Fields Method tackles this whereas the constant stress approach does not, leading to some biased results.

\subsection{Transverse compression}

\subsubsection{Experimental description}

The second test that was performed consisted in a compression transverse to the axis of the specimen, in order to investigate its possible anisotropy. Two wood plates are used to apply the load. The whole set-up is fitted in a standard electromechanical testing machine. The camera was the same as that used for the longitudinal test. An initial correlation window of 64 with a step of 8 led to an array of $73 \times 251$ measurement points. The test consisted in 13 load steps. Fig. 3 shows the initial undeformed specimen with the box marking the area of investigation and axes, while the right hand side figure shows the final stage of deformation. The strains were calculated in the same way as for the longitudinal test, with a radius of 8 .

\subsubsection{Poisson's ratio identification}

The same virtual fields have been used as for the longitudinal test. However, here, the situation is slightly different because the active area (see Fig. 3) does not encompass the whole specimen length. Therefore, zero virtual displacements have to be prescribed over the whole specimen boundary. This is already the case for the optimized virtual field but the manually defined one has to be adapted. Now, it becomes:

$$
\left\{\begin{array}{l}
u_{x}^{*}=y(y-b) x(x-L) \\
u_{y}^{*}=0
\end{array}\right.
$$

where $b$ is the specimen width (y-direction) and $L$ the length of the active area (x-direction). Apart from the fact that the virtual field now has to bear on $u_{x}^{*}$ because the load is applied in the $y$-direction, 


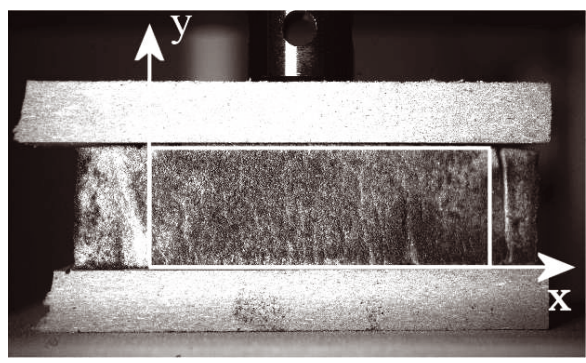

(A) Specimen at rest

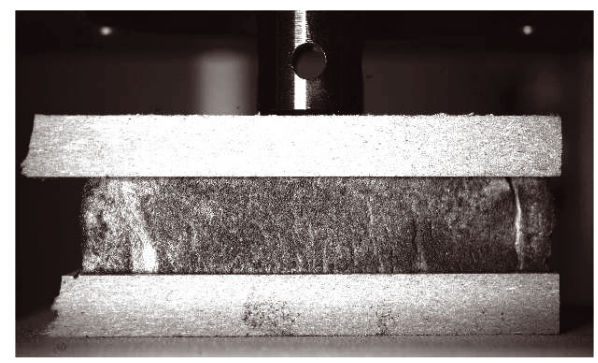

(в) Deformed specimen at final stage

Fig. 3. Auxetic specimen at rest and in final deformed state, transverse compression.

the expression is built-up so that $u_{x}^{*}=0$ for both the $\mathrm{x}$ and $\mathrm{y}$ boundaries of the active area. The resulting expression for Poisson's ratio becomes:

$$
v_{v f 1}=-\frac{\overline{(2 x-L) y(y-b) \varepsilon_{x}}+\overline{\left(y-\frac{L}{2}\right) x(x-L) \varepsilon_{s}}}{\overline{(2 x-L) y(y-b) \varepsilon_{y}}-\overline{\left(y-\frac{L}{2}\right) x(x-L) \varepsilon_{s}}}
$$

The results for the uniaxial approach, manual and optimized virtual fields are reported on Fig. 4. The first thing that can be seen on these results is that the magnitude of Poisson's ratio is much smaller than for the longitudinal configuration. There is a small auxetic effect at very low strains (here, only for the first loading stage) and then, Poisson's ratio becomes very close to zero. This suggests that the auxetic foam is anisotropic. It could be argued that because of this, the whole present approach is irrelevant because isotropy was assumed. However, with the simple test configuration and virtual fields used here, the only problem comes from assuming that the shear modulus depends on $Q_{x x}$ and $Q_{x y}$ in the previous section. In order to fully investigate this issue, the complete orthotropic set of stiffnesses should be identified which, to the best knowledge of the author, has never been attempted. This is one of the perspectives of the present paper that will require more complex tests together with the virtual fields methods.

The second observation is that the results are more scattered between the two virtual fields approach

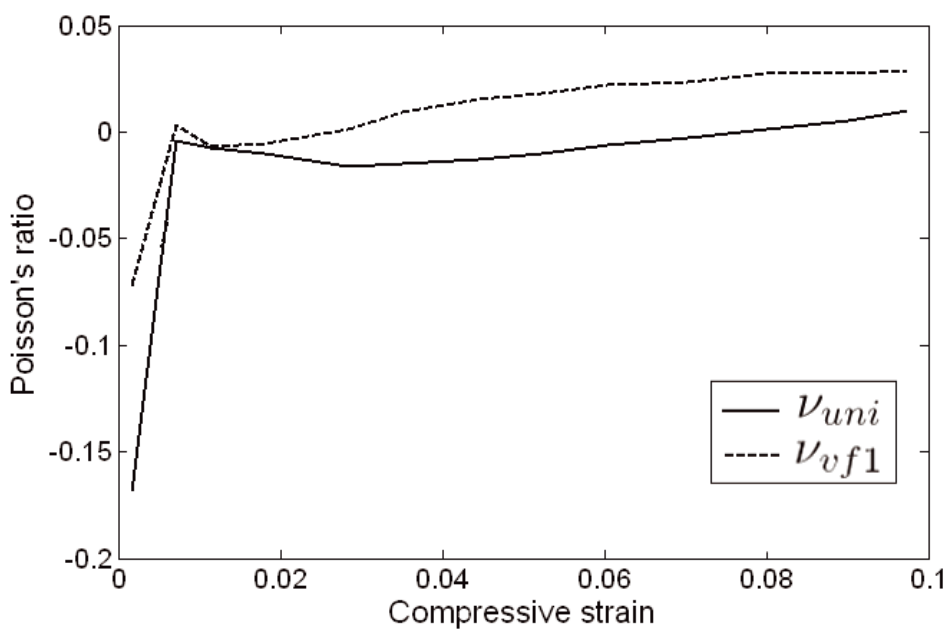

Fig. 4. Evolution of Poisson's ratio with compressive transverse deformation, uniaxial approach and manual and optimized virtual fields, auxetic transverse compression. 
than for the previous results. The reason for this appears clearly on the strain field maps shown in Fig. 5. Indeed, all $\varepsilon_{x}$ maps exhibit a pattern of positive and negative values of transverse strains, which is consistent throughout the test where areas of positive and negative strains remain the same. For the virtual fields approach, spatially weighted averages are used which means that values of Poisson's ratio obtained with different virtual fields may be different. The physical reason for this strain localization is not clear. The only certain point is that this is not a noise issue since the strains are rather high and the pattern is consistent from one load step to the other. Investigations using X-ray tomography would be useful here to study this effect in more depth. This will be attempted in the future.

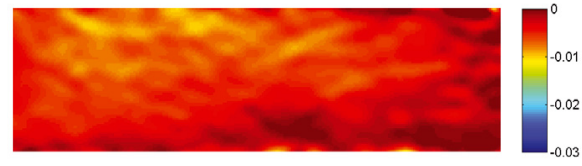

(A) $\varepsilon_{y}$ at load step 1

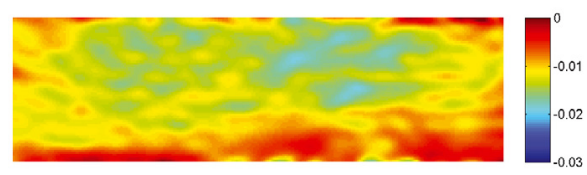

(c) $\varepsilon_{y}$ at load step 2

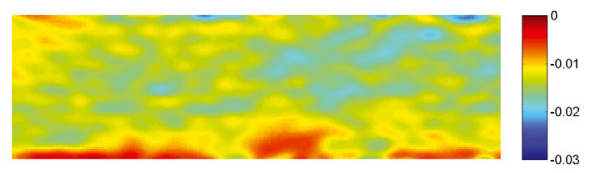

(E) $\varepsilon_{y}$ at load step 4

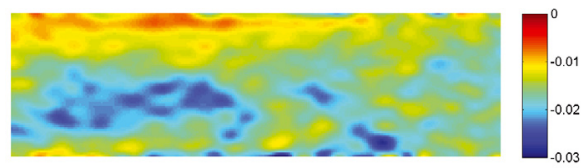

(G) $\varepsilon_{y}$ at load step 9

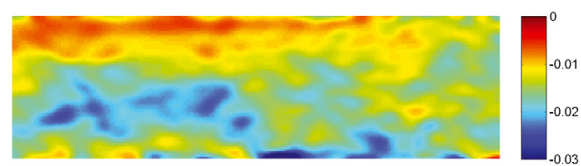

(I) $\varepsilon_{y}$ at load step 13

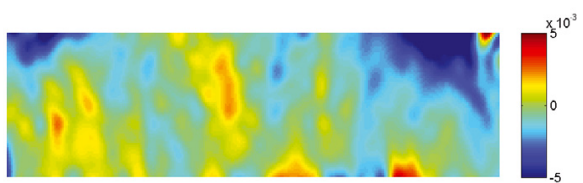

(в) $\varepsilon_{x}$ at load step 1

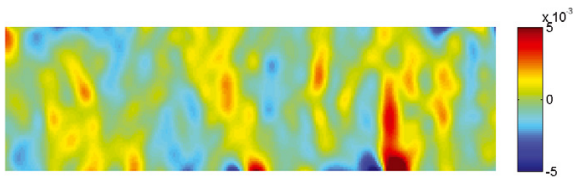

(D) $\varepsilon_{x}$ at load step 2

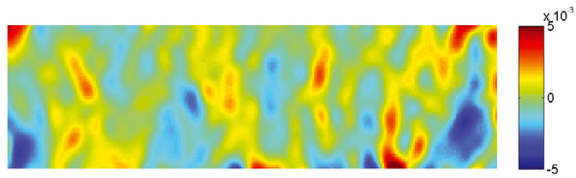

(F) $\varepsilon_{x}$ at load step 4

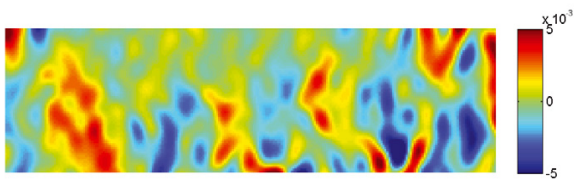

(н) $\varepsilon_{x}$ at load step 9

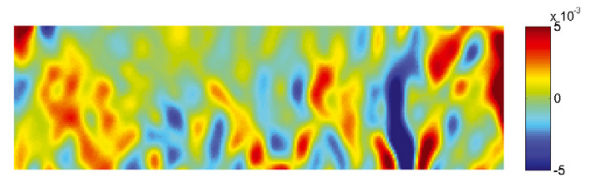

(J) $\varepsilon_{x}$ at load step 13

Fig. 5. Longitudinal and transverse strain maps all along the test, auxetic transverse compression.

\section{Conclusion and future work}

The present study has underlined the potential of full-field measurements to investigate the complex mechanical behaviour of low density polymeric foams. In particular, concerning the determination of Poisson's ratio for the auxetic foam, the following conclusions were reached. 
- The standard uniaxial approach has been shown to reach its limits in the case of the presence of significant shear strains.

- The behaviour of the auxetic foam is highly anisotropic as a consequence of its microstructure.

- The behaviour in transverse compression exhibited localized auxetic and non auxetic behaviour probably related to the local microstructure though this was not investigated in further detail. This results in a very low average Poisson's ratio.

It is clear that this area of research is still in its infancy and that the present contribution is really only a first contribution. In particular, the following points will have to be examined in the future.

- More complex tests should be performed to identify a more general constitutive model, in the same spirit as recent work on rubber [17].

- Some of the present results suggest strong local heterogeneities in Poisson's ratio (transverse test). This will have to be investigated by identifying local Poisson's ratio values and relative these to relevant microstructural parameters.

- The real challenge lies in full volume deformation measurements based on X-ray tomographic images and digital volume correlation, following some seminal work reported in [7] for instance. Such measurements coupled to effective inverse procedures such as the Virtual Fields Method have the potential to shed a new light on constitutive modeling of hyper-elastic foams.

\section{Acknowledgements}

The author gratefully acknowledges the support of the Agence Nationale de la Recherche (ANR, France) through the PHOTOFIT programme (Grant ANR-05-BLAN-0327-01). The authors are also grateful to Dr Fabrizio Scarpa and Mr Matteo Bianchi(ACCIS, University of Bristol, UK) for providing the standard and auxetic foam specimens tested in this paper and to Dr François Hild (LMT Cachan, France) for providing the "Correli" 2D correlation software. The tests were performed by Dr BaoQiao Guo (now at Tsinghua University, Beijing, China).

\section{References}

1. Y. Wang, A.C. no, International Journal of Solids and Structures 39, 3777 (2002)

2. R. Guastavino, P. Göransson, Polymer Testing 26, 711 (2007)

3. H. Jin, W.Y. Lu, S. Scheffel, T. Hinnerichs, M. Neilsen, International Journal of Solids and Structures 44, 6930 (2007)

4. J. Kinney, G. Marshall, S. Marshall, D. Haupt, Journal of Applied Polymer Science 80, 1746 (2001)

5. J. Elliott, A. Windle, J. Hobdell, G. Eeckhaut, R. Oldman, W. Ludwig, E. Boller, P. Cloetens, J. Baruchel, Journal of Materials Science 37, 1547 (2002)

6. S. McDonald, N. Ravirala, P. Withers, A. Alderson, Scripta Materialia 60, 232 (2008)

7. S. Roux, F. Hild, P. Viot, D. Bernard, Composites Part A 39, 1253 (2008)

8. C. Smith, R. Wootton, K. Evans, Experimental Mechanics 39(4), 356 (1999)

9. R. Widdle, A. Bajaj, P. Davies, International Journal of Engineering Science 46, 31 (2008)

10. M. Grédiac, F. Pierron, S. Avril, E. Toussaint, Strain 42, 233 (2006)

11. F.S. A. Bezazi, International Journal of Fatigue 29, 922 (2007)

12. F. Scarpa, P. Pastorino, A. Garelli, S. Patsias, M. Ruzzene, Physica Status Solidi B 242(3), 681 (2005)

13. M. Bianchi, F. Scarpa, C. Smith, Journal of Materials Science 43(17), 5851 (2008)

14. F. Pierron, Journal of Strain Analysis for Engineering Design (2010), accepted

15. F. Hild, B. Raka, M. Baudequin, S. Roux, F. Cantelaube, Applied Optics 41, 6815 (2002)

16. S. Avril, P. Feissel, F. Pierron, P. Villon, European Journal of Computational Mechanics 17(5-7), 857 (2008)

17. N. Promma, B. Raka, M. Grédiac, E. Toussaint, J.B.L. Cam, X. Balandraud, F. Hild, International Journal of Solids and Structures 46(3-4), 698 (2009) 